\title{
Comparison of the proliferation, migration and angiogenic properties of human amniotic epithelial and mesenchymal stem cells and their effects on endothelial cells
}

\author{
QIANQIAN WU ${ }^{1}$, TAO FANG $^{2}, \mathrm{HONGXIN} \mathrm{LANG}^{3}, \mathrm{MIN} \mathrm{CHEN}^{1}, \mathrm{PING} \mathrm{SHI}^{4}, \mathrm{XINING} \mathrm{PANG}^{3}$ and GUOXIAN QI ${ }^{1}$ \\ ${ }^{1}$ Department of Geriatric Cardiology and Department of Cardiology, The First Affiliated Hospital of China Medical University, \\ Shenyang, Liaoning 110001; ${ }^{2}$ Department of Orthopedic Surgery, The Shengjing Hospital of China Medical University, \\ Shenyang, Liaoning 110004; ${ }^{3}$ Department of Stem Cells and Regenerative Medicine, Key Laboratory of Cell Biology, \\ Chinese Ministry of Public Health and Key Laboratory of Medical Cell Biology, Chinese Ministry of Education, \\ China Medical University, Shenyang, Liaoning 110013; ${ }^{4}$ Department of General Practice, \\ The First Affiliated Hospital of China Medical University, Shenyang, Liaoning 110001, P.R. China
}

Received December 3, 2016; Accepted February 6, 2017

DOI: $10.3892 /$ ijmm.2017.2897

\begin{abstract}
In vivo studies have shown that amnion-produced growth factors participate in many diseases that involve angiogenesis, re-epithelialization and immunomodulation. Although human amniotic epithelial cells (hAECs) and human amniotic mesenchymal stem cells (hAMSCs) can be obtained from amniotic membranes, there is little information regarding their biological differences. The aim of the present study was to isolate and characterize cells from human amnions, to investigate the biological potential and behavior of these cells on the function of endothelial cells in vivo and in vitro and to examine variations in the expression profile of growth factors in different human amnion-derived cell types. Amnion fragments were enzymatically digested into two cell fractions, which were analyzed by mesenchymal and epithelial cell markers. Human aortic endothelial cells (hAoECs) were cultured with conditioned medium (CdM) collected from hAECs or hAMSCs. We used scratch and Transwell assays to evaluate migration ability; Cell Counting Kit-8 (CCK-8) and cell cycle analysis to evaluate proliferation ability; and a Matrigel tube formation assay to
\end{abstract}

Correspondence to: Dr Guoxian Qi, Department of Geriatric Cardiology and Department of Cardiology, The First Affiliated Hospital of China Medical University, 155 Nanjing North Street, Heping, Shenyang, Liaoning 110001, P.R. China

E-mail: qigx2002@medmail.com.cn

Dr Xining Pang, Department of Stem Cells and Regenerative Medicine, Key Laboratory of Cell Biology, Chinese Ministry of Public Health and Key Laboratory of Medical Cell Biology, Chinese Ministry of Education, China Medical University, 77 Puhe Street, Shenbei New District, Shenyang, Liaoning 110013, P.R. China E-mail: pangxining@126.com

Key words: human amniotic epithelial cells, human amniotic mesenchymal stem cell, paracrine, angiogenesis evaluate angiogenesis ability. To detect expression of angiogenesis-related genes, qPCR and enzyme-linked immunosorbent assay (ELISA) analyses were conducted. As stem cells, hAECs and hAMSCs all expressed the stem cell markers SSEA-4, OCT-4 and SOX-2. CdM obtained from hAECs promoted cell migration; CdM obtained from hAMSCs promoted cell proliferation; CdM obtained from hAECs and hAMSCs both promoted angiogenesis in hAoECs. Amnion-derived cells secreted significant amounts of angiogenic factors including HGF, IGF-1, VEGF, EGF, HB-EGF and bFGF, although differences in the cellular expression profile of these soluble factors were observed. Our results highlight that human amniotic epithelial and mesenchymal stem cells, which showed differences in their soluble factor secretion and angiogenic functions, could be ideal cell sources for regenerative medicine.

\section{Introduction}

Appendages of the fetus, included the amnion, are normally discarded after delivery as medical waste. A large quantity of human amniotic epithelial and mesenchymal stem cells can be obtained non-invasively from the amniotic membrane, which represents an advantageous source of cells for cell therapy (1).

In vivo studies have previously reported the therapeutic potential of stem cells using various animal models including hindlimb ischemia $(2,3)$, wound healing $(4,5)$ and myocardial infarction $(6,7)$. However, in many cases, the frequency of stem cell engraftment and the number of newly generated adult cells, either by transdifferentiation or cell fusion, appear to be too low to explain the significant improvement described $(8,9)$. Meanwhile, tissue concentrations of proteins, including vascular endothelial growth factor (VEGF) and basic fibroblast growth factor (bFGF) are increased in the injured areas treated with stem cells (10). There is a growing body of evidence supporting the hypothesis that paracrine mechanisms mediated by factors released by pluripotent stem cells play an essential role in the reparative process $(11,12)$. This paracrine 
effect renders these cells an attractive therapeutic source for regenerative medicine.

Stem cells may be beneficial in various cell-therapeutic approaches where they function by promoting the survival of endothelial cells $(13,14)$, the stabilization of pre-existing vessels (15), and the revascularization of ischemic tissues $(2,3)$. Given that the natural response to tissue repair is such a complex process, many growth factors may be involved. Thus, a great deal of interest has arisen in angiogenetic factors found in stem cells, such as hepatocyte growth factor (HGF), epidermal growth factor (EGF), heparin binding EGF like growth factor (HB-EGF) and insulin growth factor-1 (IGF-1), and the paracrine effects which are significantly related to the angiogenesis of endothelial cells (3,16-18).

The aim of the present study was: i) to isolate and characterize cells from human amnions; ii) to investigate the biological potential and behavior of these cells in regards to the function of endothelial cells in vivo and in vitro; and iii) to examine the variations in the expression profile of growth factors in different human amnion-derived cell types.

\section{Materials and methods}

Ethics. The amnion samples discarded after Caesarean sections were collected from the Department of Obstetrics and Gynecology, The First at Hospital of China Medical University (Shenyang, China). This study was approved by the Ethics Committee of the First Affiliated Hospital of China Medical University. Written informed consent was obtained from all of the patients prior to their participation. C57BL/6J mice were obtained from the Experimental Animal Centre of China Medical University. All experiments and animal care were approved by the Ethics Committee of the First Affiliated Hospital of China Medical University.

\section{Cells and cell culture}

Human aorta endothelial cells (hAoECs). hAoECs were purchased from ScienCell (Carlsbad, CA, USA) and cells at passage 4-6 were used for in vitro experiments. The cells were cultured in Endothelial Basal Media-2 (EBM-2) with $5 \%$ fetal bovine serum (FBS) and Endothelial Cell Growth Supplement (ECGS) (EGM-2; ScienCell).

Human amniotic epithelial cells (hAECs). Primary cell culture was performed as described previously (5). Briefly, amnions were manually separated and washed with phosphate-buffered saline (PBS) supplemented with $100 \mathrm{U} / \mathrm{ml}$ penicillin and streptomycin. Amnions were then incubated with $0.25 \%$ trypsin solution for $30 \mathrm{~min}$. This process was repeated three times. Supernatants were collected and centrifuged for $5 \mathrm{~min}$ at $1,000 \mathrm{rpm}$ to obtain a cell pellet. Those cells were plated on a culture flask (designated as hAEC P0) in Dulbecco's modified Eagle's medium (DMEM; HyClone, Logan, UT, USA), and $100 \mathrm{U} / \mathrm{ml}$ penicillin and streptomycin. In this study, hAECs at passage 2-3 were used.

Human amniotic mesenchymal stem cells (hAMSCs). The amnion tissue was cut into small pieces, and then incubated with $1 \mathrm{mg} / \mathrm{ml}$ collagenase IV (Sigma-Aldrich, St. Louis, MO, USA) and $0.1 \mathrm{mg} / \mathrm{ml}$ DNase (Takara Bio, Inc., Shiga, Japan) at $37^{\circ} \mathrm{C}$ for $20 \mathrm{~min}$. FBS was then added to stop digestion, and supernatants were filtered through a cell strainer $(200 \mu \mathrm{m})$ and centrifuged for $5 \mathrm{~min}$ at $1,000 \mathrm{rpm}$. Cells were plated on a culture flask (designated as hAMSC P0) in DMEM containing 10\% FBS (both from HyClone), $10 \mathrm{mmol} / \mathrm{ml} \mathrm{FGF-2}$ (PeproTech, Rocky Hill, NJ, USA), and $100 \mathrm{U} / \mathrm{ml}$ penicillin and streptomycin. The culture medium was changed $48 \mathrm{~h}$ later. In this study, hAMSCs at passages 3-6 were used for the functional experiment.

The differentiation capacity of amniotic cells. hAECs (passages 2) and hAMSCs (passage 3) were tested for their ability to differentiate into osteocytes, chondrocytes and adipocytes.

To induce differentiation into osteocytes, the cells were cultured in osteocyte differentiation medium: $1 \mu \mathrm{M}$ dexamethasone, $50 \mu \mathrm{g} / \mathrm{ml} \mathrm{L}$-ascorbate, and $10 \mathrm{mM} \beta$-glycerophosphate (Sigma-Aldrich) in DMEM supplemented with 10\% FBS. After 14 days of differentiation, the cells were fixed and stained with Alizarin Red S (Cyagen, Guangzhou, China).

To induce differentiation into adipocytes, the cells were cultured with adipocyte differentiation medium: $0.5 \mathrm{mM}$ 3-isobutyl-1-methyl xanthine, $1 \mu \mathrm{M}$ dexamethasone, $200 \mu \mathrm{M}$ indomethacin, and $10 \mu \mathrm{g} / \mathrm{ml}$ insulin (Sigma-Aldrich) in DMEM supplemented with $10 \%$ FBS. After 14 days of differentiation, the cells were stained with Oil Red O (Cyagen).

To induce differentiation into chondrocytes, the cells were cultured with chondrocyte differentiation medium: $0.1 \mu \mathrm{M}$ dexamethasone, $50 \mu \mathrm{g} / \mathrm{ml} \mathrm{L-ascorbate,} 100 \mu \mathrm{g} / \mathrm{ml}$ sodium pyruvate (Sigma-Aldrich), and $10 \mathrm{ng} / \mathrm{ml}$ transforming growth factor (TGF)- $\beta 1$ (PeproTech) in DMEM supplemented with $10 \%$ FBS. After 14 days of differentiation, the cells were stained with Alcian blue (Cyagen).

Flow cytometry and immunofluorescence of cells. Flow cytometry and immunofluorescence were used to identify the characteristics of the cells and detect stem cell-related cell surface markers. For flow cytometry, the cells $\left(10^{6}\right.$ cells $\left./ 100 \mu \mathrm{l}\right)$ were collected and incubated with monoclonal phycoerythrin (PE)-conjugated antibodies for CD29 (cat. no. 303003), CD31 (cat. no. 303105), CD34 (cat. no. 343505), CD44 (cat. no. 338807), CD45 (cat. no. 368509), CD49d (cat. no. 304303), CD73 (cat. no. 344003), CD90 (cat. no. 32810), CD105 (cat. no. 323205), HLA-DR (cat. no. 307605), SSEA-4 (cat. no. 330405), SOX-2 (cat. no. 656103) and OCT-4 (cat. no. 653703) (BioLegend, San Diego, CA, USA) for $30 \mathrm{~min}$ on ice. Appropriate isotype-matched antibodies were used as negative controls (BD Biosciences, San Jose, CA, USA). Data from 10,000 viable cells were acquired. List mode files were analyzed by FCS Express software (BD Biosciences). For immunofluorescence, cells growing on the glass slide were stained with anti-cytokeratin 19 (cat. no. ab52625, 1:200; Abcam, Cambridge, MA, USA) and secondary antibody (cat. no. A-11034, 1:200; Alexa 488, donkey anti-rabbit; Life Technologies, Carlsbad, CA, USA). Nuclei were stained with DAPI (Beyotime, Shanghai, China). Cells on the glass slide were photographed using an inverted fluorescence microscope (Carl Zeiss, Oberkochen, Germany).

Preparation of conditioned medium. To generate conditioned medium (CdM), hAoECs, hAMSCs and hAECs were cultured with EGM-2. After the cells had reached $\sim 50-60 \%$ confluence 
$\left(\sim 4 \times 10^{5}\right.$ cells in $25 \mathrm{~cm}^{2}$ flask), cultures were gently rinsed three times with PBS and the medium was replaced with EGM-2 or EBM-2. After 48 h, the CdM (EGM-2) obtained from each plate was then collected, pooled for each cell type, centrifuged at $1,000 \mathrm{x} \mathrm{g}$ for $5 \mathrm{~min}$, filtered $(0.2 \mu \mathrm{m})$ to remove cellular debris, stored at $-80^{\circ} \mathrm{C}$ and supernatants were used as CdM-hAoEC, CdM-hAEC and CdM-hAMSC for cell assays. Positive control, non-conditioned medium (non-CdM) was generated in the same way as above, except that no cells were cultured in the plates. Batches of $2 \mathrm{X}$ concentrated $\mathrm{CdM}$ (EBM-2) were also prepared for the in vivo Matrigel plug assay. In this way, a final concentration of $1 \mathrm{X} \mathrm{CM}$ after 1:1 dilution in Matrigel was obtained.

Cell viability assays. For the growth curves of hAECs and hAMSCs, cells $\left(5 \times 10^{3} /\right.$ well) were plated in 96-well plates with EGM-2. Cells were cultured for 7 days, and cell proliferation was measured using Cell Counting Kit-8 (CCK-8; Dojindo, Kumamoto, Japan) every day according to the manufacturer's protocol. For determining the effect of $\mathrm{CdM}$ on endothelial cell viability, hAoECs were cultured in EGM-2 without FBS for $24 \mathrm{~h}$ to arrest mitosis. Then, hAoECs $\left(2 \times 10^{4} /\right.$ well $)$ were plated in 96-well plates, the medium was replaced with CdM-hAoEC (control), CdM-hAEC, CdM-hAMSC and EGM-2 (positive control). Cells were cultured for $24 \mathrm{~h}$, after which hAoEC proliferation was measured using the CCK-8 (Dojindo). In brief, cells were incubated with CCK-8 for $1.5 \mathrm{~h}$ at $37^{\circ} \mathrm{C}$. The staining intensity in the medium was measured by determining the absorbance at $450 \mathrm{~nm}$.

Cell cycle analysis. The effect of CdM on cell cycle distribution was determined by flow cytometry. Briefly, hAoECs were treated with different CdM for $24 \mathrm{~h}$. Cells were suspended, washed with PBS, centrifuged, and fixed in $70 \%$ ethanol at $-20^{\circ} \mathrm{C}$ overnight. Cells were then resuspended in $500 \mu \mathrm{l}$ of dyeing buffer containing $10 \mu \mathrm{l}$ RNase A and $25 \mu 1$ PI (Beyotime). Cells were incubated in the dark for $30 \mathrm{~min}$ at room temperature. A total of $1 \times 10^{4}$ cells were subjected to cell cycle analysis using a flow cytometer (BD Biosciences).

Scratch wound closure assay. hAoECs $(500,000$ cells/insert) were plated in 6-well plates, and at $80-90 \%$ confluence, at $12 \mathrm{~h}$ after plating, a scratch of $\sim 0.5 \mathrm{~mm}$ was created using a sterile pipette tip. Each well was washed twice with PBS and then the cell culture medium was either replaced with CdM-hAoEC (control), CdM-hAEC, CdM-hAMSC or EGM-2 (positive control). Cell migration into the scratch was photographed at 0,6 and $24 \mathrm{~h}$ using an inverted microscope (Olympus, Tokyo, Japan). Results were analyzed with Image-Pro Plus software 6.0 (IPP; Media Cybernetics, Inc., Rockville, MD, USA). The results are presented as the percentage of wound healing, which was calculated as follows: [Wound area (initial) - Wound area (final)]/Wound area (initial) x 100\% (5).

Transwell migration assay. Cell migration assays were performed using inserts with $8-\mu \mathrm{m}$ pore-sized membranes in a 24-well plate (Corning Costar, Lowell, MA, USA). CdM-hAoEC (control), CdM-hAEC, CdM-hAMSC and EGM-2 (positive control) were placed in the bottom chamber. hAoECs were resuspended in serum-free EGM-2, transferred onto the filter of the insert $(50,000$ cells/insert $)$ and incubated at $37^{\circ} \mathrm{C}$ in $5 \% \mathrm{CO}_{2}$ for 6 or $24 \mathrm{~h}$. Non-migratory cells were removed from the upper side of the filter. Migratory cells at the bottom side of the filter were fixed with paraformaldehyde (PFA), stained with hematoxylin and eosin (H\&E) and photographed. Cells were counted from eight randomly selected regions/well.

Matrigel tube formation assay. To evaluate the tube formation potential, hAoECs were seeded with each CdM derived from different cells, at a concentration of $2.5 \times 10^{4}$ cells/well in growth factor-reduced basement membrane matrix gel (Matrigel; BD Biosciences)-coated 96-well plates. After 6, 24 and $48 \mathrm{~h}$ of incubation, representative fields were photographed using inverted microscopy (Olympus), and branching points from each sample were examined with Image-Pro Plus software 6.0.

Matrigel plug assays. The CdM (EBM-2) was mixed with $250 \mu \mathrm{l}$ of liquid Matrigel-reduced growth factor (BD Matrigel $356230)$ at a ratio of $1: 1$ at $4^{\circ} \mathrm{C}$. Mice (8-weeks old) received a total of $500 \mu \mathrm{l}$ of this mixture subcutaneously in the dorsal region, generating Matrigel plugs when warmed to body temperature. Plugs were recovered 1 week later.

qPCR assay for growth factor and cytokine detection. Total RNA was extracted, using TRIzol reagent (Invitrogen, Carlsbad, CA, USA), from hAECs and hAMSCs cultured with EGM-2 complete medium. RNA concentration was determined by NanoDrop ND-1000 (NanoDrop Technologies, Wilmington, DE, USA). cDNA was synthesized using PrimeScript $^{\mathrm{TM}}$ RT reagent (Takara Bio, Inc.). Reactions were performed using the SYBR PrimeScript RT-PCR kit (Takara Bio, Inc.) with an ABI 7500 Sequence Detection system (Applied Biosystems, Foster City, CA, USA). As an internal control, the $\beta$-actin level was quantified in parallel with the target genes. Normalization and fold-changes were calculated using the $\Delta \Delta \mathrm{Cq}$ method. The primers used for realtime PCR are the following: 5'-CTGTCTAATGCCCTG GAGCC/ACGCGAGTCTGTGTTTTTGC-3' for VEGFA; 5'-TCAGCCAGCAGATGGGAATG/TCAGGGCTGTATGG GCAAAG-3' for EGF; 5'-GGCTGTACTGCAAAAACGGG/ TAGCTTGATGTGAGGGTCGC-3' for bFGF; 5'-CAATGC CTCTGGTTCCCCTT/TGTTCCCTTGTAGCTGCGTC-3' for HGF; 5'-AGTTCTCTCGGCACTGGTGA/TAGCAGCTG GTCCGTGGATA-3' for HB-EGF; 5'-ATCAGCAGTCTT CCAACCCA/GAGATGCGAGGAGGACATGG-3' for IGF-1; 5'-AGGATTCCTATGTGGGCGAC/ATAGCACAGCCTGG ATAGCAA-3' for $\beta$-actin.

Enzyme-linked immunosorbent assay (ELISA) detection of angiogenetic growth factors. Conditioned medium from hAoECs, hAECs and hAMSCs were collected after $48 \mathrm{~h}$ incubation. The concentration of cytokines in the different CdM was measured using sandwich ELISA kits (VEGFA, EGF, bFGF, HGF, HB-EGF and IGF-1; R\&D Systems, Minneapolis, MN, USA). After media were collection, the cells were counted. ELISA values were corrected for total cell numbers. Positive control-conditioned medium was also assayed. 
A

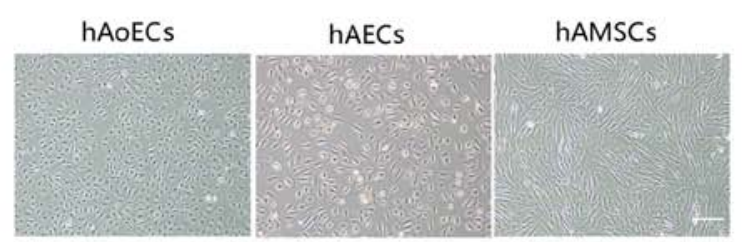

C
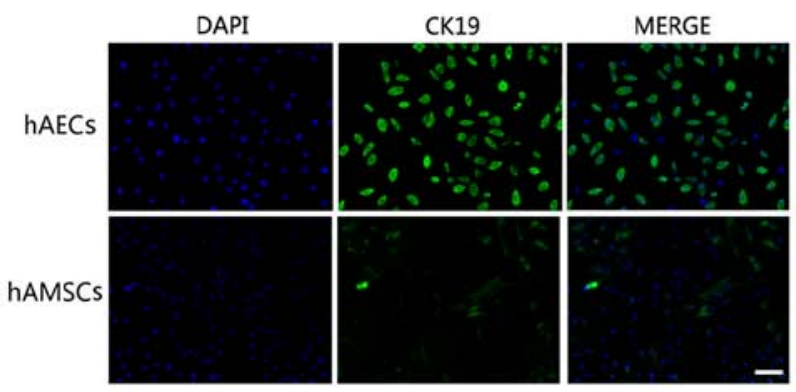
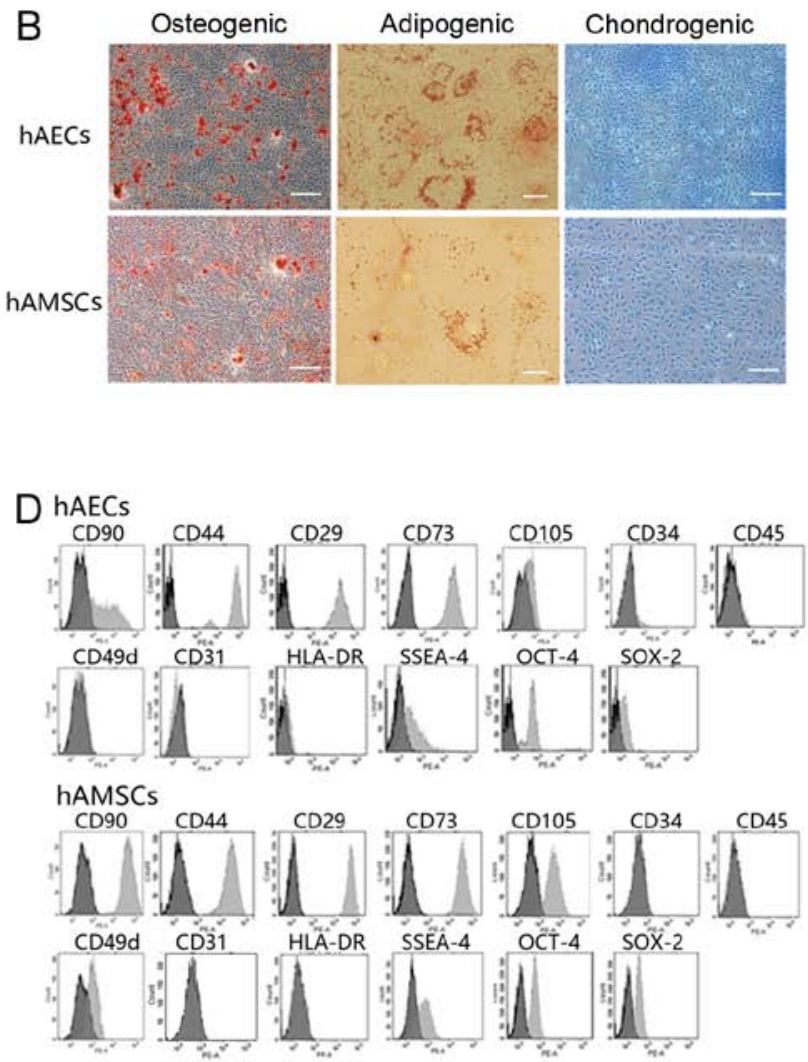

Figure 1. Characterization of human amniotic epithelial and mesenchymal stem cells. (A) Representative images of human aortic endothelial cells (hAoECs) (passage 4) and cultured cells obtained from human amniotic epithelial (passage 2) and mesenchymal (passage 4) stem cells. Scale bar, 200 $\mu$ m. (B) Differentiation of amniotic cells into osteocytes (scale bars, $200 \mu \mathrm{m}$ ), adipocytes (scale bars, $20 \mu \mathrm{m}$ ) and chondrocytes (scale bars, $200 \mu \mathrm{m}$ ). Cells cultured under osteogenic adipogenic or chondrogenic culture conditions were stained for calcium deposits with alizarin red staining, lipid droplets with Oil Red O staining or proteoglycans with Alcian blue staining, respectively. (C) Immunofluorescence was conducted to evaluate cytokeratin 19 expression in human amniotic epithelial cells (hAECs) and human amniotic mesenchymal stem cells (hAMSCs). Scale bar, $100 \mu \mathrm{m}$. (D) FACS analysis of cell markers of hAECs and hAMSCs (light gray bars) compared with isotype-matched antibodies (dark gray bars).

Statistical analysis. All experiments were performed 3 times on amniotic cells and with CdM from 3 different donors. The data are shown as the means \pm SDs. Comparisons between groups were analyzed using t-test. Comparisons of parameters for more than three groups were made by one-way analysis of variance (ANOVA) followed by the Bonferroni test. All statistical analyses were performed using SPSS 17.0 computer software. P-values $<0.05$ were considered to indicate statistically significant differences.

\section{Results}

Characterization of $h A E C s$ and hAMSCs. hAECs exhibited a cobblestone-like morphology, similar to hAoECs. Cultured hAMSCs showed a spindle fibroblast-like morphology (Fig. 1A). Flow cytometry and immunofluorescence revealed the expression of surface markers. hAECs were positive for CK19, CD29, CD44, CD73, CD90 and CD105, but were negative for CD31, CD34, CD45 and CD49d. hAMSCs were positive for CD29, CD44, CD49d, CD73, CD90 and CD105, but were negative for CK19, CD31, CD34 and CD45 (Fig. 1C and D). In addition, amniotic cells were all negative for HLA-DR, indicating that these cells possess low immunogenicity. To confirm the stem cell characteristics of hAECs and hAMSCs, we performed FACS analysis using embryonic stem and germ cell markers. Amniotic cells were all found to express SSEA-4, SOX2 and OCT-4 (Fig. 1D). These results are consistent with previously reported data $(1,19,20)$. In addition, hAECs and hAMSCs could differentiate into osteocytes, adipocytes and chondrocytes, as demonstrated by positive Alizarin Red, Oil Red O and Alcian blue staining, respectively (Fig. 1B), which indicated that cultured amniotic cells possess stem cell characteristics.

Culture medium from hAMSCs enhances proliferation ability. We tested the growth kinetics of hAECs and hAMSCs. Our data showed that when cultured with EGM-2, cells obtained from the same amnion had a similar proliferation ability/day (Fig. 2A). Since the proliferation of hAoECs is an important aspect of angiogenesis, we compared the proliferation ability of hAoECs when stimulated with different CdMs. The CCK-8 assay revealed that hAoECs cultured in CdM-hAMSC showed enhanced proliferation compared with the other CdMs, even the positive control EGM-2. However, there was no significant difference in $\mathrm{hAoEC}$ proliferation between CdM-hAEC and CdM-hAoEC (Fig. 2B). In order to further confirm the effect of $\mathrm{CdM}$ on the proliferation ability of hAoECs, we examined the effect of CdM on cell cycle distribution using flow cytometry. Compared to the basal level $(21.61 \pm 1.54 \%)$, hAoECs treated with CdM-hAMSC led to a marked increase in the number of cells in the $S$ phase $(30.56 \pm 1.91 \%)$ (Fig. 2C). There was no statistical difference, 
A

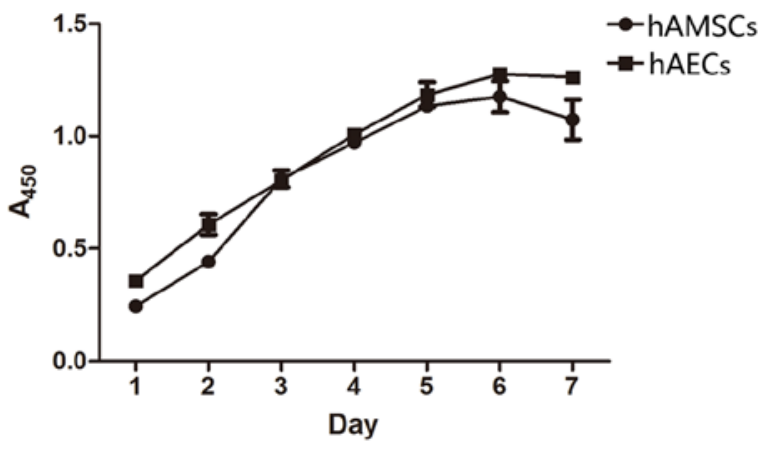

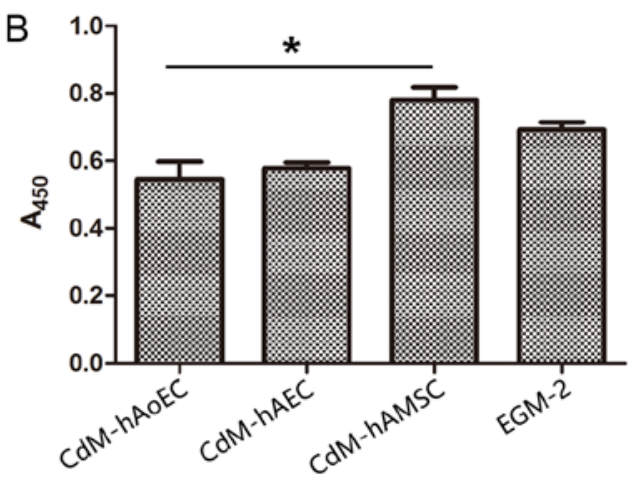
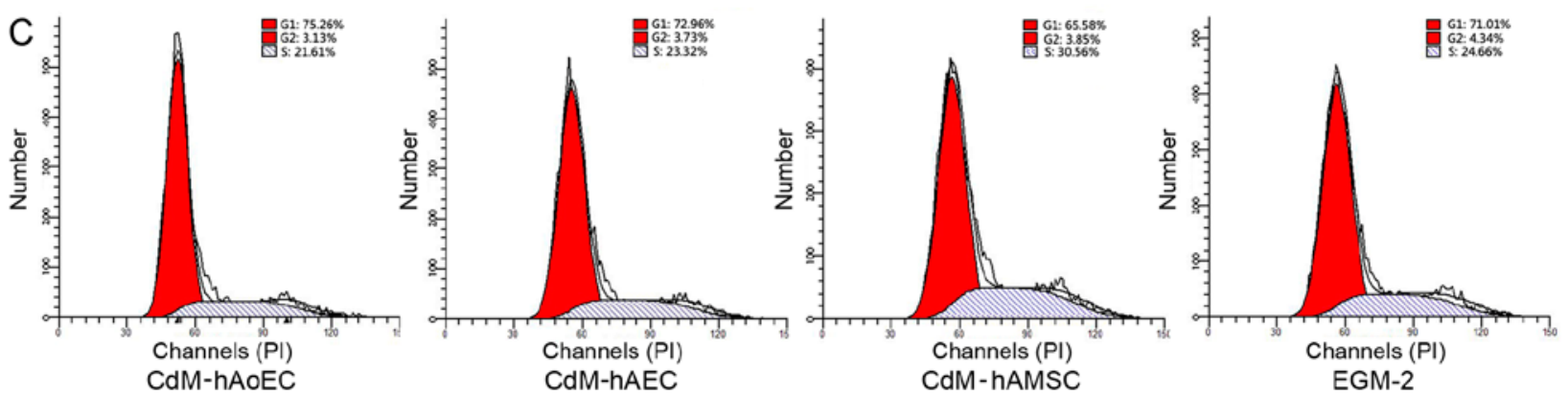

Figure 2. Effect of conditioned medium (CdM) on the proliferation of human aortic endothelial cells (hAoECs). (A) Comparison of the growth kinetics of amniotic cells. (B) CCK-8 assay was conducted to evaluate the cytoprotective effect of the CdMs which were collected after incubation for $48 \mathrm{~h}$. CdM-hAMSC significantly improved the cytoprotective effect compared with the other groups. (C) Representative flow cytometric analysis of the cell cycle of hAoECs cultured with CdMs. Date represent the mean \pm SD of there independent experiments. " $\mathrm{P}<0.05$. CdM-hAoEC (control), CdM from hAoECs; CdM-hAEC, CdM from human amniotic epithelial cells; CdM-hAMSC, CdM from human amniotic mesenchymal stem cells.

however, when hAoECs were treated with CdM-hAEC or CdM-hAoEC.

Culture medium from hAECs enhances migration ability. In order to examine whether CdMs exhibited biological effects relevant to hAoEC migration, we compared the effects of the different CdMs on migration by means of scratch and Transwell assays. The images showed that hAoEC migration into the scratch wound area was accelerated when cultured with CdM-hAEC (75.86 $\pm 3.06 \%$ CdM-hAEC vs. 29.16 $\pm 5.12 \%$ CdM-hAMSC; $\mathrm{P}<0.05,20.11 \pm 7.04 \%$ CdM-hAoEC; n=3/group) (Fig. 3C). In Transwell cell migration assays, our results revealed that CdM-hAEC significantly increased the rate of hAoEC migration compared with CdM-hAMSC and CdM-hAoEC (184.01 \pm 33.66 CdM-hAEC vs. 58.82 \pm 23.99 CdM-hAMSC; $\mathrm{P}<0.05,45.20 \pm 30.04$ CdM-hAoEC; n=3/group) (Fig. 3A).

Angiogenesis assay: network formation. The Matrigel assay is a commonly used method to evaluate network formation by endothelial cells and was applied to investigate whether induced $\mathrm{CdM}$ were also involved in forming networks. After $6 \mathrm{~h}$, the addition of CdM-hAEC to hAoECs supported the formation of network-like structures in the Matrigel assay, to a greater extent than CdM-hAoEC. After $24 \mathrm{~h}$, the addition of CdM-hAEC to hAoECs still supported the formation of network-like structures, but this was not statistically significant. While networks formed by endothelial cells in CdM-hAoEC had disintegrated after $48 \mathrm{~h}$, networks formed by hAoECs cultured with CdM-hAEC and CdM-hAMSC were still stable (Fig. 4). To examine the angiogenic potential of CdM in vivo, we used the murine Matrigel plug assay.
At 1 week after implantation, the Matrigel plug containing CdM-hAEC and CdM-hAMSC formed a blood vessel network connected with the host vasculature. These vessels contained blood. while there was no blood vessel in the negative group (Fig. 5).

Expression of angiogenesis-specific mRNAs in hAMSCs and $h A E C s$. We used qRT-PCR to investigate the angiogenic-related mRNA expression in the hAMSCs and hAECs. hAECs showed significantly higher expression of the angiogenic gene HB-EGF, which was 71-fold higher than the expression in the hAMSCs. Notably, hAMSCs had a higher expression of bFGF, HGF and IGF-1 compared to these levels in the hAECs ( $>30$-fold higher). In addition, EGF and VEGFA, angiogenic factors that are pivotal in neovascularization, were expressed to a similar extent in the hAECs and hAMSCs (Fig. 6A).

Expression of angiogenic proteins in CdMs. Collected $\mathrm{CdMs}$ were analyzed for the presence of angiogenic proteins using an ELISA kit (Fig. 6B). The EGM-2 value was taken as a background level. Compared with $\mathrm{CdM}$ collected from hAoECs (HB-EGF, 205.2 $\pm 25.3 \mathrm{pg} / 10^{6}$ cells; EGF, $-72.9 \pm 43.26 \mathrm{pg} / 10^{6}$ cells; bFGF, $-106.4 \pm 33.3 \mathrm{pg} / 10^{6}$ cells; HGF, 89.9 $2.8 \mathrm{pg} / 10^{6}$ cells), amniotic cells secreted higher levels of angiogenic factors. In line with the different mRNA expression levels, compared with CdM-hAMSC (HB-EGF, $326.8 \pm 25.4 \mathrm{pg} / 10^{6}$ cells; bFGF, $97.0 \pm 2.8 \mathrm{pg} / 10^{6}$ cells; HGF, $1024.5 \pm 98.3 \mathrm{pg} / 10^{6}$ cells), CdM-hAEC had a higher level of the proangiogenic factor HB-EGF $\left(518.0 \pm 53.5 \mathrm{pg} / 10^{6}\right.$ cells $)$, and lower levels of bFGF $\left(-30.0 \pm 14.4 \mathrm{pg} / 10^{6}\right.$ cells $)$ and HGF $\left(472.1 \pm 49.8 \mathrm{pg} / 10^{6}\right.$ cells $)$. There was no difference between 

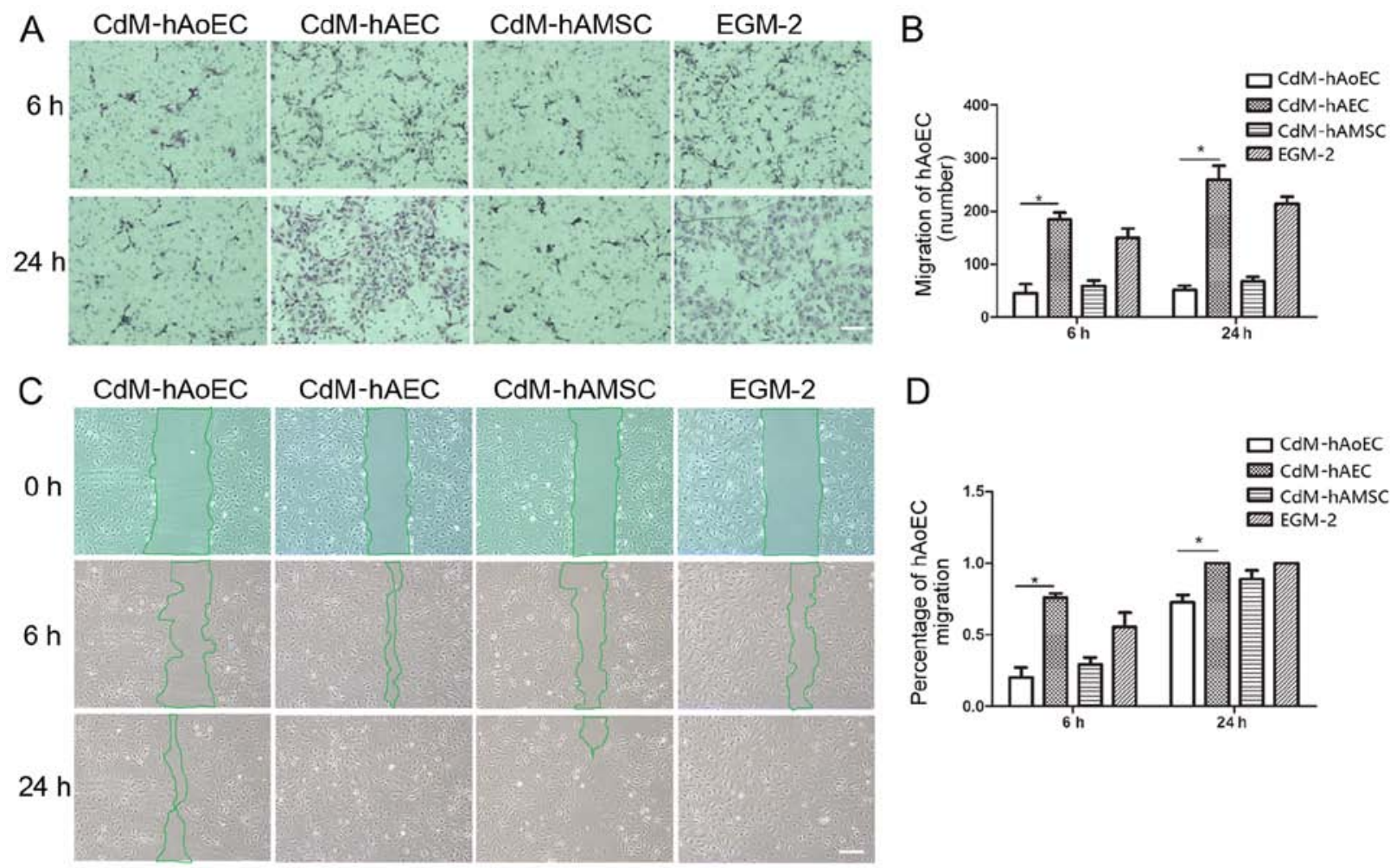

Figure 3. Effect of conditioned medium (CdM) on the migration of human aortic endothelial cells (hAoECs). (A) Images of hematoxylin and eosin-stained membranes in the Transwell hAoEC migration assay after a 6- and 24-h coculture with CdM. (B) Rate of hAoEC movement. Results indicate that hAoEC migration into the bottom chamber was accelerated in the presence of CdM-hAEC. Date represent the mean $\pm \mathrm{SD}$ of there independent experiments. ${ }^{*} \mathrm{P}<0.05$ (C) Migration of hAoECs into the scratch wound after 0,6 and $24 \mathrm{~h}$ of culture with CdM in the scratch wound assay. (D) Rate of hAoEC movement after 6 and $24 \mathrm{~h}$. The rate of movement was signifiantly greater for hAoECs cultured with CdM-hAEC compared with the other groups. Scale bar, $200 \mu \mathrm{m}$. CdM-hAoEC (control), CdM from hAoECs; CdM-hAEC, CdM from human amniotic epithelial cells; CdM-hAMSC, CdM from human amniotic mesenchymal stem cells.
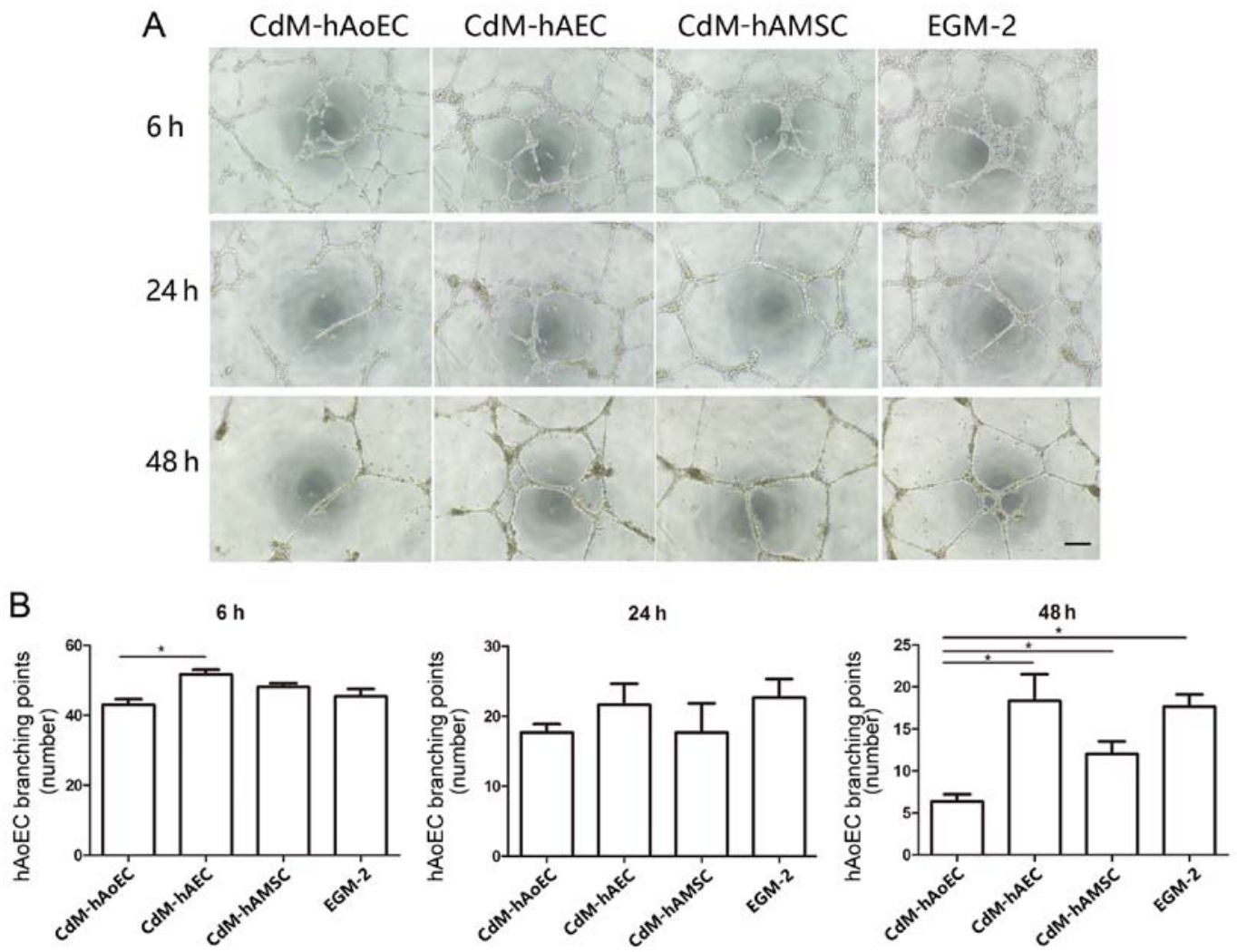

Figure 4. Matrigel tube formation analysis in vitro. (A) Representative images of Matrigel tube formation using conditioned medium (CdM) at 6, 24 and $48 \mathrm{~h}$. CdM-hAoEC was used as a negative control. Scale bar, $200 \mu \mathrm{m}$. (B) Representation of the branching point number of hAoECs. The number was significantly higher in the CdM-hAEC and CdM-hAMSC group. "P<0.05. CdM-hAoEC, CdM from human aortic endothelial cells; CdM-hAEC, CdM from human amniotic epithelial cells; CdM-hAMSC, CdM from human amniotic mesenchymal stem cells. 
A

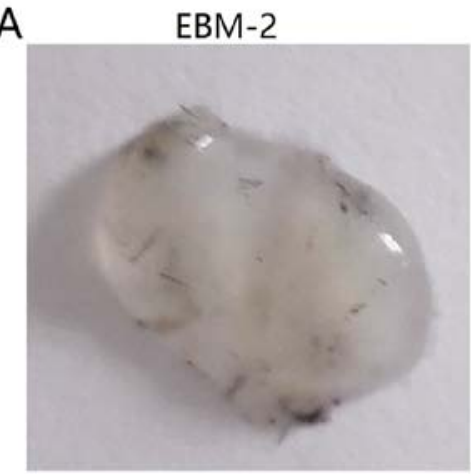

B

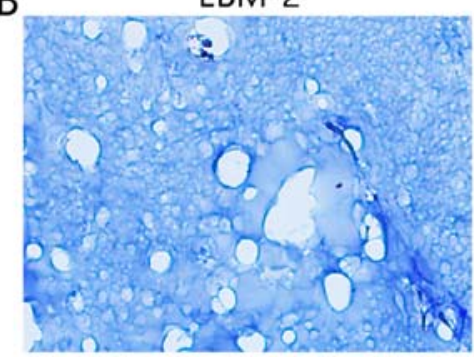

CdM(EBM-2) hAEC

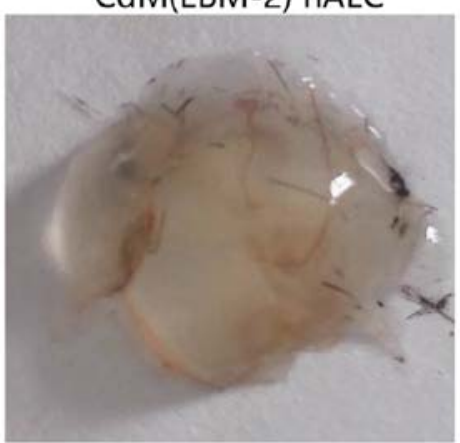

CdM(EBM-2) hAEC

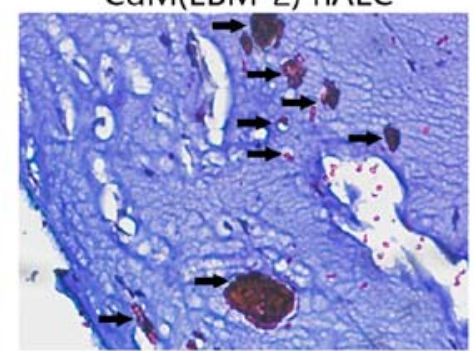

$\mathrm{CdM}(\mathrm{EBM}-2)$ hAMSC
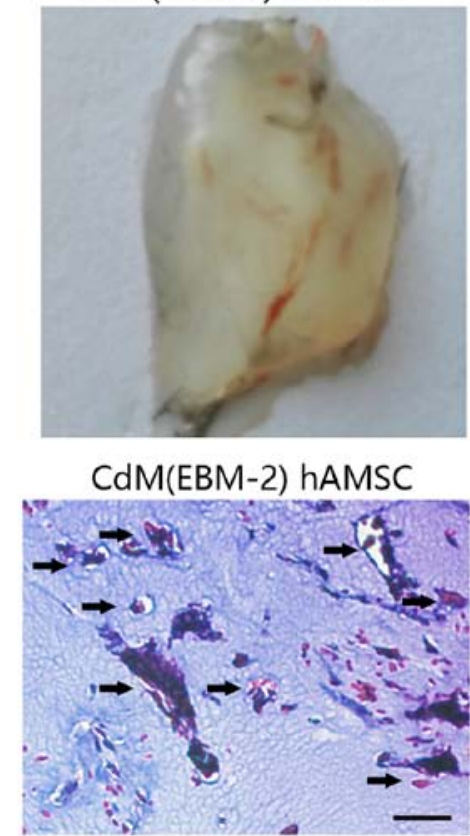

Figure 5. Matrigel tube formation analysis in vivo. (A) Gross anatomy of freshly removed Matrigel plugs with conditional medium 1 week after implantation. (B) Masson trichrome staining of plug sections of EBM-2, CdM(EBM-2) hAEC and CdM(EBM-2) hAMSC-treated mice. CdM from both amniotic cells promoted blood vessels formation in vivo. Scale bar, $50 \mu \mathrm{m}$. CdM, conditioned medium; EBM-2, Endothelial Basal Medium-2; hAEC, human amniotic epithelial cell; hAMSC, human amniotic mesenchymal stem cell.
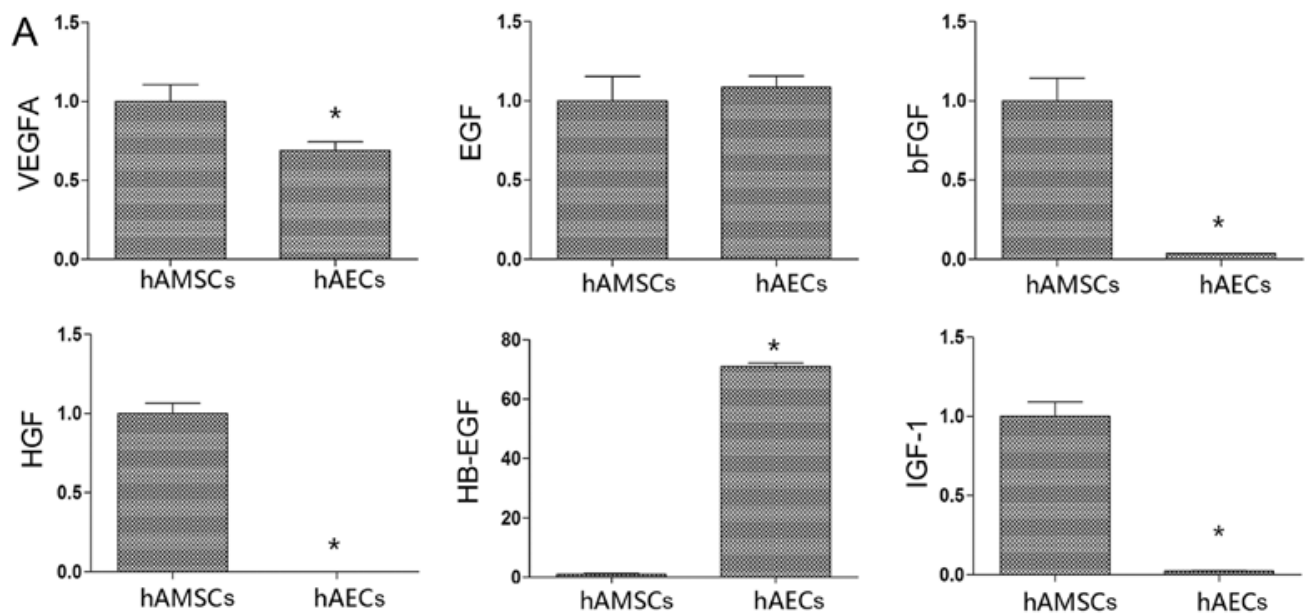

B

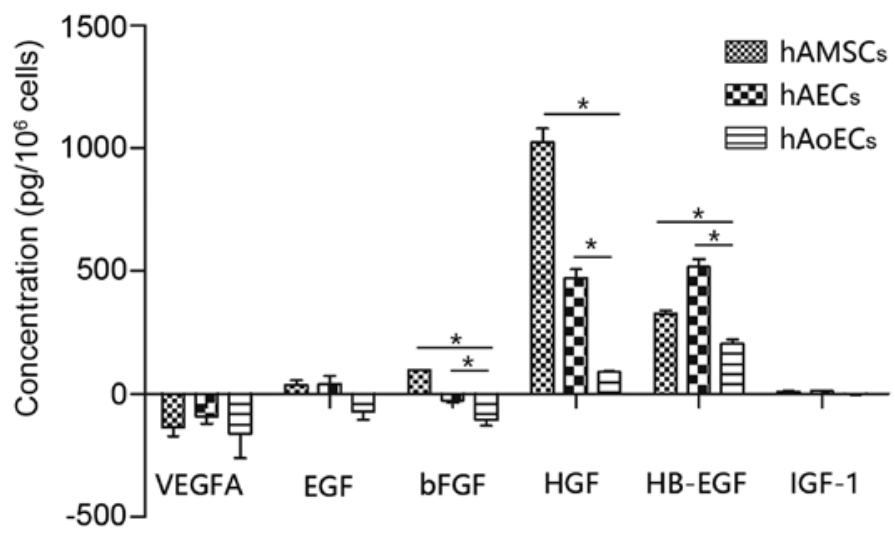

Figure 6. Angiogenetic growth factor expression profile. (A) qRT-PCR was conducted to evaluate multiple angiogenic gene levels in hAECs and hAMSCs. All individual values were normalized to $\beta$-actin ( $\mathrm{n}=3 /$ group). ${ }^{*} \mathrm{P}<0.05$. (B) The concentration of vascular endothelial growth factor (VEGF), epidermal growth factor (EGF), basic fibroblast growth factor (bFGF), hepatocyte growth factor (HGF), heparin binding EGF like growth factor (HB-EGF), insulin growth factor-1 (IGF-1) inconditional medium was measured by enzyme-linked immunosorbent assay (ELISA). "P<0.05. hAECs, human amniotic epithelial cells; hAMSCs, human amniotic mesenchymal stem cells. 
hAECs and hAMSCs in regards to VEGFA expression $\left(-135.1 \pm 61\right.$ and $-91.1 \pm 51.9 \mathrm{pg} / 10^{6}$ cells, respectively) or EGF expression ( $38.3 \pm 29.7$ and $41.5 \pm 57.9 \mathrm{pg} / 10^{6}$ cells, respectively). Notably, although there was an obvious difference in IGF-1 mRNA levels, there was no difference in IGF-1 protein levels $\left(10.5 \pm 3.8\right.$ and $12.8 \pm 2.1 \mathrm{pg} / 10^{6}$ cells $)$.

\section{Discussion}

Many studies have previously demonstrated the therapeutic potential of stem cells using animal models including wound healing $(4,5)$, limb ischemia $(2,3)$, and myocardial infarction $(6,7)$. The proliferation, migration and angiogenic properties of endothelial cells are important in the revascularization of ischemic tissue and the reperfusion of myocardial infarction. While recent studies have revealed the angiogenic properties of human amniotic membrane and mesenchymal stem cells $(16,21)$, information concerning hAECs is rare, and comparative studies of the biological effects between hAECs and hAMSCs are lacking. Therefore, in the present study, we reported that amniotic cells possess high biological potential for endothelial cells and we compared the differences in the cellular function and biological properties between these cells. The main findings of this study were: i) hAECs and hAMSCs display similar growth kinetics, and express stem cell markers; ii) CdM-hAEC significantly promoted endothelial cell migration, CdM-hAMSC promoted endothelial cell proliferation, and they both promoted the stabilization of angiogenesis; iii) there was high expression of HB-EGF in hAECs; and iv) high expression of bFGF and HGF in hAMSCs. To our knowledge, this is the first study to compare the beneficial effects of hAECs and hAMSCs on endothelial cell function.

We are convinced that human amniotic cells are an attractive source for cell therapy because they are free from ethical concern, a large number of cells can be obtained, and they display low immunogenicity, consistent with a previous study (22). We can also obtain a large number of cells from a small piece of amniotic membrane, thus ensuring that there are abundant cells available for future clinical therapy. hAECs and hAMSCs displayed similar growth kinetics when cultured with EGM-2; and when we collected CdMs, the number of hAECs and hAMSCs were found to be similar, thus making the comparison of growth factors in CdMs more reasonable. After xenogeneic transplantation into neonatal swine and rats, hAMSCs engraft without immunosuppression (23-25). In accordance with these studies, amniotic cells were negative for HLA-DR, which indicated that they have low immunogenicity, which can be taken as an advantage for in vivo therapy. OCT-3/4, SOX-2 and SSEA-4 are pluripotent markers that are commonly expressed by stem cells (26). Consistent with previous studies $(19,26)$, hAMSCs and hAECs also differentiated toward mesodermal lineages (osteogenic, chondrogenic, and adipogenic), and expressed stem cell markers, SSEA-4, SOX-2 and OCT-4, which indicates they have a high ability of pluripotency and self-renewal.

In vivo studies demonstrated that there were few differentiated cells being tested and the tissue concentrations of growth factors were significantly increased in injured areas treated with stem cells in transplanted models $(10,27)$. We believe that the ability of amniotic cells to stimulate regenerative effects is mainly induced via paracrine routes. Furthermore, this theory is confirmed by several studies, which showed that conditioned medium from MSCs promoted the recovery of myocardial infarction $(6,7,12)$. Therefore, we collected $\mathrm{CdM}$ from amniotic cells, and tested the biological effect of migration, proliferation and angiogenesis on hAoECs. The collection time of $48 \mathrm{~h}$ was chosen based on the literature (15). As an in vitro assay of cell migration, we performed scratch and Transwell experiments. CdM-hAEC markedly affected hAoEC migration compared with CdM-hAMSC and CdM-hAoEC. These results are consistent with in vivo research showing that hAECs promote epithelialization and wound healing (4). CdM-hAMSC had a positive effect on hAoECs, as shown by enhanced viability and proliferation ability in the cell cycle distribution assay. CdMs from hAECs and hAMSCs stabilized blood vessel network formation in vitro and stimulated blood vessel formation in vivo. These results are consistent with research showing that MSCs from bone marrow promote angiogenesis and support blood vessel formation (3).

It has been shown that stem cells secrete a broad variety of cytokines, chemokines, and growth factors, which may potentially be involved in regenerative medicine $(3,6,28)$. The molecular processes leading to angiogenesis involve mediators such as EGF, HB-EGF, VEGF, bFGF, HGF, IGF-1 and others, which lead to cell migration, proliferation, vessel formation and maturation (3,16-18,29-32). The present study demonstrates that numerous arteriogenic cytokines are released by MSCs (11). This study demonstrated that hAECs highly express HB-EGF; HAMSCs secreted significantly larger amounts of HGF and bFGF, and gene results were confirmed using ELISA assays. The difference in expression of IGF-1 mRNA was not immediately obvious when tested by ELISA assay, perhaps due to epigenetic regulation. We assume that these differences in the cytokine expression profile could reflect the angiogenic and cytoprotective properties of amniotic cells, as we observed differences in their effects on hAoECs in our conditionedmedium analysis.

In the present study, considering that amniotic cells play a role in the microenvironment of endothelial cells, and that EGM-2 functions in a similar manner, we chose EGM-2 for conditioned medium collection to coordinate culture medium between different cells. Cytokines not only have individual effects, but one cytokine may potentiate (or inhibit) the effect of another, i.e. having a synergistic or antagonistic relationship (33). Determining the nature and mechanism(s) of the paracrine soluble molecules involved in CdM-mediated angiogenesis stabilization is obviously a challenge for all researchers in the field.

In conclusion, this study demonstrated the differential effects of amniotic cells on the function of hAoECs, via paracrine angiogenetic-related growth factors. Therefore, both cell types may provide a convenient source for clinical therapy.

\section{Acknowledgements}

This study was supported by the grants from the National Basic Research Program of China (grant no. 2012CB518103), the National Natural Science Foundation of China (grant no. 81450017), the Science and Technology Department of Liaoning Province (grant no. 2013020200-206), the Science 
and Technology Department of Liaoning Province (grant no. 2014305012), the Science and Technology Bureau of Shenyang City (grant nos. F15-157-1-00), the Science and Technology Planning Project of Shenyang (grant no. F14-201-4-00).

\section{References}

1. Ilancheran S, Michalska A, Peh G, Wallace EM, Pera M and Manuelpillai U: Stem cells derived from human fetal membranes display multilineage differentiation potential. Biol Reprod 77: 577-588, 2007.

2. Kim SW, Zhang HZ, Kim CE, An HS, Kim JM and Kim MH: Amniotic mesenchymal stem cells have robust angiogenic properties and are effective in treating hindlimb ischaemia. Cardiovasc Res 93: 525-534, 2012.

3. Kinnaird T, Stabile E, Burnett MS, Lee CW, Barr S, Fuchs S and Epstein SE: Marrow-derived stromal cells express genes encoding a broad spectrum of arteriogenic cytokines and promote in vitro and in vivo arteriogenesis through paracrine mechanisms. Circ Res 94: 678-685, 2004.

4. Yoshida Y, Tanaka S, Umemori H, Minowa O, Usui M, Ikematsu N, Hosoda E, Imamura T, Kuno J, Yamashita T, et al: Negative regulation of BMP/Smad signaling by Tob in osteoblasts. Cell 103: 1085-1097, 2000.

5. Liu X, Wang Z, Wang R, Zhao F, Shi P, Jiang Y and Pang X: Direct comparison of the potency of human mesenchymal stem cells derived from amnion tissue, bone marrow and adipose tissue at inducing dermal fibroblast responses to cutaneous wounds. Int J Mol Med 31: 407-415, 2013.

6. Fidelis-de-Oliveira P, Werneck-de-Castro JPS, Pinho-Ribeiro V, Shalom BC, Nascimento-Silva JH, Costa e Souza RH, Cruz IS Rangel RR, Goldenberg RC and Campos-de-Carvalho AC: Soluble factors from multipotent mesenchymal stromal cells have antinecrotic effect on cardiomyocytes in vitro and improve cardiac function in infarcted rat hearts. Cell Transplant 21: 1011-1021, 2012.

7. Timmers L,Lim SK, Hoefer IE, Arslan F, Lai RC, van Oorschot AA, Goumans MJ, Strijder C, Sze SK, Choo A, et al: Human mesenchymal stem cell-conditioned medium improves cardiac function following myocardial infarction. Stem Cell Res (Amst) 6: 206-214, 2011.

8. Balsam LB, Wagers AJ, Christensen JL, Kofidis T, Weissman IL and Robbins RC: Haematopoietic stem cells adopt mature haematopoietic fates in ischaemic myocardium. Nature 428: 668-673, 2004.

9. Nygren JM, Jovinge S, Breitbach M, Säwén P, Röll W, Hescheler J, Taneera J, Fleischmann BK and Jacobsen SE: Bone marrow-derived hematopoietic cells generate cardiomyocytes at a low frequency through cell fusion, but not transdifferentiation. Nat Med 10: 494-501, 2004.

10. Kinnaird T, Stabile E, Burnett MS, Shou M, Lee CW, Barr S, Fuchs S and Epstein SE: Local delivery of marrow-derived stromal cells augments collateral perfusion through paracrine mechanisms. Circulation 109: 1543-1549, 2004.

11. Konala VBR, Mamidi MK, Bhonde R, Das AK, Pochampally R and Pal R: The current landscape of the mesenchymal stromal cell secretome: A new paradigm for cell-free regeneration. Cytotherapy 18: 13-24, 2016.

12. Yamaguchi S, Shibata R, Yamamoto N, Nishikawa M, Hibi H, Tanigawa T, Ueda M, Murohara T and Yamamoto A: Dental pulp-derived stem cell conditioned medium reduces cardiac injury following ischemia-reperfusion. Sci Rep 5: 16295, 2015.

13. König J, Huppertz B, Desoye G, Parolini O, Fröhlich JD, Weiss G, Dohr G, Sedlmayr P and Lang I: Amnion-derived mesenchymal stromal cells show angiogenic properties but resist differentiation into mature endothelial cells. Stem Cells Dev 21 : 1309-1320, 2012.

14. Sha X, Liu Z, Song L, Wang Z and Liang X: Human amniotic epithelial cell niche enhances the functional properties of human corneal endothelial cells via inhibiting P53-survivinmitochondria axis. Exp Eye Res 116: 36-46, 2013.

15. König J, Weiss G, Rossi D, Wankhammer K, Reinisch A Kinzer M, Huppertz B, Pfeiffer D, Parolini O and Lang I: Placental mesenchymal stromal cells derived from blood vessels or avascular tissues: What is the better choice to support endothelial cell function? Stem Cells Dev 24: 115-131, 2015.
16. Yamahara K, Harada K, Ohshima M, Ishikane S, Ohnishi S, Tsuda H, Otani K, Taguchi A, Soma T, Ogawa H, et al: Comparison of angiogenic, cytoprotective, and immunosuppressive properties of human amnion- and chorion-derived mesenchymal stem cells. PLoS One 9: e88319, 2014.

17. Yotsumoto F, Tokunaga E, Oki E, Maehara Y, Yamada $\mathrm{H}$, Nakajima K, Nam SO, Miyata K, Koyanagi M, Doi K, et al: Molecular hierarchy of heparin-binding EGF-like growth factorregulated angiogenesis in triple-negative breast cancer. Mol Cancer Res 11: 506-517, 2013.

18. Kim SW, Zhang HZ, Guo L, Kim JM and Kim MH: Amniotic mesenchymal stem cells enhance wound healing in diabetic NOD/SCID mice through high angiogenic and engraftment capabilities. PLoS One 7: e41105, 2012.

19. Miki T, Lehmann T, Cai H, Stolz DB and Strom SC: Stem cell characteristics of amniotic epithelial cells. Stem Cells 23: 1549-1559, 2005.

20. Parolini O, Alviano F, Bagnara GP, Bilic G, Bühring HJ, Evangelista M, Hennerbichler S, Liu B, Magatti M, Mao N, et al: Concise review: Isolation and characterization of cells from human term placenta: Outcome of the first international Workshop on Placenta Derived Stem Cells. Stem Cells 26: 300-311, 2008.

21. Grzywocz Z, Pius-Sadowska E, Klos P, Gryzik M, Wasilewska D, Aleksandrowicz B, Dworczynska M, Sabalinska S, Hoser G, Machalinski B, et al: Growth factors and their receptors derived from human amniotic cells in vitro. Folia Histochem Cytobiol 52: 163-170, 2014.

22. Alviano F, Fossati V, Marchionni C, Arpinati M, Bonsi L, Franchina M, Lanzoni G, Cantoni S, Cavallini C, Bianchi F, et al: Term Amniotic membrane is a high throughput source for multipotent Mesenchymal Stem Cells with the ability to differentiate into endothelial cells in vitro. BMC Dev Biol 7: 11, 2007.

23. Manochantr S, U-pratya Y, Kheolamai P, Rojphisan S, Chayosumrit M, Tantrawatpan C, Supokawej A and Issaragrisil S: Immunosuppressive properties of mesenchymal stromal cells derived from amnion, placenta, Wharton's jelly and umbilical cord. Intern Med J 43: 430-439, 2013.

24. Magatti M, De Munari S, Vertua E, Gibelli L, Wengler GS and Parolini O: Human amnion mesenchyme harbors cells with allogeneic T-cell suppression and stimulation capabilities. Stem Cells 26: 182-192, 2008.

25. Magatti M, De Munari S, Vertua E, Nassauto C, Albertini A, Wengler GS and Parolini O: Amniotic mesenchymal tissue cells inhibit dendritic cell differentiation of peripheral blood and amnion resident monocytes. Cell Transplant 18: 899-914, 2009.

26. Fatimah SS, Tan GC, Chua K, Fariha MMN, Tan AE and Hayati AR: Stemness and angiogenic gene expression changes of serial-passage human amnion mesenchymal cells. Microvasc Res 86: 21-29, 2013

27. Gnecchi M, He H, Liang OD, Melo LG, Morello F, Mu H, Noiseux N, Zhang L, Pratt RE, Ingwall JS, et al: Paracrine action accounts for marked protection of ischemic heart by Akt-modified mesenchymal stem cells. Nat Med 11: 367-368, 2005.

28. Caplan AI and Dennis JE: Mesenchymal stem cells as trophic mediators. J Cell Biochem 98: 1076-1084, 2006.

29. Yamakawa H, Muraoka N, Miyamoto K, Sadahiro T, Isomi M, Haginiwa S, Kojima H, Umei T, Akiyama M, Kuishi Y, et al: Fibroblast growth factors and vascular endothelial growth factor promote cardiac reprogramming under defined conditions. Stem Cell Reports 5: 1128-1142, 2015.

30. Chen QH, Liu AR, Qiu HB and Yang Y: Interaction between mesenchymal stem cells and endothelial cells restores endothelial permeability via paracrine hepatocyte growth factor in vitro. Stem Cell Res Ther 6: 44, 2015.

31. Barrientos S, Stojadinovic O, Golinko MS, Brem $\mathrm{H}$ and Tomic-Canic M: Growth factors and cytokines in wound healing. Wound Repair Regen 16: 585-601, 2008.

32. Chen L, Tredget EE, Wu PY and Wu Y: Paracrine factors of mesenchymal stem cells recruit macrophages and endothelial lineage cells and enhance wound healing. PLoS One 3: e1886, 2008.

33. Yang Y, Chen QH, Liu AR, Xu XP, Han JB and Qiu HB: Synergism of MSC-secreted HGF and VEGF in stabilising endothelial barrier function upon lipopolysaccharide stimulation via the Rac1 pathway. Stem Cell Res Ther 6: 250, 2015. 